\title{
Compliance with Pregnancy Prevention Recommendations for Isotretinoin in Estonia in 2012-2016
}

\author{
Anneli Uusküla $^{1}$ (1) $\cdot$ Heti Pisarev ${ }^{1} \cdot$ Katrin Kurvits $^{2} \cdot$ Ott Laius $^{2} \cdot$ Made Laanpere $^{3}$. \\ Maia Uusküla ${ }^{2}$
}

Published online: 22 May 2018

(c) The Author(s) 2018

\begin{abstract}
Background Isotretinoin is an effective treatment for severe acne; no alternative treatment has an equal therapeutic effect. The teratogenic effects of isotretinoin can be avoided, and numerous recommendations and regulations are in force to minimize the risk of pregnancy during treatment. Objectives To describe isotretinoin prescription patterns for women aged 15-45 years, assess the concomitancy of isotretinoin and contraceptive use, and determine the rate of potential isotretinoin-exposed pregnancies in Estonia.

Methods This retrospective, nationwide, population-based, cohort study derived data from national health insurance databases and included female patients aged 15-45 years in Estonia for whom one or more prescriptions for isotretinoin were dispensed between 2012 and 2016. The main outcome was the proportion of women who used systemic isotretinoin and had a concomitant record of (hormonal or intrauterine) contraception use covering the isotretinoin treatment period when pregnancy is contraindicated.

Results Of the 2792 women aged 15-45 years filling an isotretinoin prescription, $15.7 \%$ (95\% CI 14.4-17.1) had full and $13.9 \%$ (95\% CI 12.7-15.3) partial (not covering the whole period during which pregnancy is contraindicated) contraceptive coverage. The risk for potential isotretinoin-exposed pregnancy was 3.6 (95\% CI 2.0-7.0) per 1000 treated women over the 5-year observation period.
\end{abstract}

Anneli Uusküla

anneli.uuskula@ut.ee

1 Department of Family Medicine and Public Health, University of Tartu, Ravila 19, 50411 Tartu, Estonia

2 Agency of Medicines, 50411 Tartu, Estonia

3 Department of Obstetrics and Gynaecology, University of Tartu, Tartu, Estonia
The odds for full coverage with effective contraception increased with the age of the patient, with the duration of isotretinoin treatment and over the period of observation. Conclusion Our study adds to the existing literature documenting limited compliance with pregnancy prevention programs for isotretinoin-containing products, and calls for program assessment to identify whether new measures should be taken or whether weaknesses in policy or implementation can be corrected.

\section{Key Points}

This nationwide population-based study conducted in Estonia documented very low effective contraceptive coverage during isotretinoin treatment among women of reproductive age.

We also documented a very low risk of unintended pregnancies in the group of women on isotretinoin and partially or not covered by contraception, perhaps due to abstinence, a contraceptive choice that has not yet been quantified in this population.

A multinational evaluation of pregnancy prevention programmes is needed to detect the underlying factors contributing to the wide disparities in contraceptive coverage.

Policymakers and public health professionals can use these risk factors to address weaknesses in prevention programme policy or implementation, or both, to reduce the number of potentially exposed pregnancies. 


\section{Introduction}

Isotretinoin is an effective treatment for severe acne; no alternative treatment has an equal therapeutic effect [1]. However, isotretinoin is also teratogenic in humans, thus numerous recommendations and regulations are in force to minimize the risk of pregnancy during treatment. Following a European Commission decision in October 2003, each member state implemented a defined Pregnancy Prevention Programme (PPP) for isotretinoin-containing products, e.g. use of effective contraception before and throughout the treatment; medically supervised pregnancy testing before, during and after the treatment; limiting prescriptions should a 30-day supply; and dispensing within 7 days of prescribing [2]. Although the basic requirements for PPP are harmonised across EU member states, there are several marketing authorisation holders for isotretinoin, and their educational materials may differ in some details (e.g. wording or layout). This may lead to discrepancies in the PPP messaging between countries. A study conducted in 2009 among the pharmaceutical regulatory agencies of member states found that $95 \%$ of responding countries $(n=22)$ had implemented PPP, and in $82 \%$ all required elements had been incorporated [3]. However, $73 \%$ reported a total of 143 isotretinoin-exposed pregnancies since implementation of the harmonized PPP. Similarly, a systematic review of isotretinoin prescribing in Europe suggested a failure of PPP implementation [4].

A study assessing concomitant use of isotretinoin and contraceptives before and after a similar program (iPledge) was introduced in the USA found isotretinoin prescriptions declined; however, no changes were found in contraceptive use during treatment [5]. In the 12 years since Estonia implemented a PPP, three isotretinoin-exposed pregnancies were reported to the Agency of Medicines.

However, aside from these reports of exposed pregnancies, few national or subnational studies have assessed the concomitant use of contraceptives with isotretinoin therapy and the rate of pregnancies among isotretinointreated women (Netherlands [3, 6], Canada [7]).

This study advances our understanding of populationbased behaviours related to pregnancy prevention guideline adherence by: (1) describing isotretinoin prescription patterns among women aged 15-45 years, (2) assessing the relationship between isotretinoin and contraceptive use, and (3) determining the rate of pregnancy during isotretinoin treatment in Estonia.

\section{Materials and Methods}

This retrospective, nationwide, population-based, cohort study included female patients aged 15-45 years in Estonia for whom one or more prescriptions for isotretinoin were dispensed between 2012 and 2016.

\subsection{Data Source}

We obtained data from the Estonian Health Insurance Fund (EHIF). Since the early 2000s, the EHIF has maintained a complete record of in- and out-patient healthcare services provided and prescriptions issued. In the EHIF, both the International Nonproprietary Name (INN) and the Anatomical Therapeutic Chemical (ATC) Classification Systems allow medications and purchase amounts to be extracted. In addition, the date of prescription, purchase and prescribing doctor (including location and specialty) are available. International Classification of Diseases (ICD10) diagnostic codes are also available. In Estonia, the requirement for a physician's prescription to obtain isotretinoin is strictly enforced. There are three reimbursement rates for isotretinoin for those insured by the EHIF: $90 \%$ (for those aged $\leq 16$ years, $75 \%$ (in case the prescription is issued by dermatovenerologist or by other physicians continuing the treatment initiated by dermatovenerologists) and 50\% (all physicians).

As of 31 December 2014, the EHIF had 1232,819 members (individuals covered with insurance) representing 93.9\% of the Estonian population [8]. It should be noted that prescriptions for uninsured individuals are also captured, although their medications are not reimbursed.

\subsection{Data}

Data were obtained to (1) identify females receiving isotretinoin; (2) describe contraceptives concomitant to isotretinoin used by these women; and (3) determine the occurrence of isotretinoin-exposed pregnancies during the 5-year study period.

To identify individuals who had been treated with isotretinoin, we retrieved data on all prescriptions for isotretinoin (ATC D10BA01) issued to women during the period 1 January 2012-31 October 2016 from EHIF. Next, we identified contraception via prescription data for hormonal contraception, or healthcare data regarding services provided for the insertion or management of intrauterine contraceptives. The following data were abstracted for hormonal contraception: (1) intrauterine contraceptives (ATC code beginning with G02BA); (2) intravaginal contraceptives (ATC code G02BB01); and (3) hormonal contraceptives for systemic use-oral, transdermal, implants 
(ATC codes beginning with G03A) (for the period of 1 November 2011-31 December 2016).

For each prescription (isotretinoin or contraceptive) the following data were recorded: medication/device (including ATC code, trade name, amount prescribed and purchased); prescribing physician specialty; prescription date and prescription status (filled/not filled); date of filling; patient's age and delinked identifier to allow longitudinal tracking without personal identification.

Healthcare service data related to the insertion or management of intrauterine contraceptives were captured from claims by ICD 10 codes, including the date of service, patient's delinked identification, and physician specialty, as follows: Z30.1-insertion of (intrauterine) contraceptive device; Z30.5-surveillance of (intrauterine) contraceptive device during the study period (1 January 2011-31 December 2016).

Pregnancy-related healthcare services were abstracted from claims by ICD 10 codes for the study period (1 January 2012-31 October 2016) as follows: O00-O08 (pregnancy with abortive outcome), Z32.1 (pregnancy confirmed), Z33 (pregnant state, incidental), Z34 (supervision of normal pregnancy), and Z35 (high-risk pregnancy). For all the above, dates of service provision were also captured. In Estonia, abortion has been legal and accessible for a long period of time. Since 1955, women have been legally allowed to request an abortion up until the 12th week of pregnancy. Termination on medical grounds is allowed until the 22nd week of pregnancy.

\subsection{Cohort Entry and Definitions}

Female patients were included in the cohort upon filling a prescription for isotretinoin during the study period. A series of prescriptions was considered a continuous episode of drug therapy if a prescription was filled less than 61 days after the previous prescription. The episode duration was calculated as the period (in days) from the first to the last (continuous) filling date of the prescription plus 30 days. Gaps between prescriptions $>60$ days from the length of the previous prescription were considered a break in therapy (and thus ending the period). The $>60$-day gap was chosen to account for Estonian regulations, which state that for chronic diseases and/or prolonged treatment, the prescription should be made for an amount of drug needed for at least 2 months of treatment [9]. Only the first episode of isotretinoin treatment was included in the analysis. The daily dose taken (mg/daily) was calculated by dividing the total amount of isotretinoin (in milligrams) purchased by the duration of the period (in days).

\subsection{Use of Contraceptives}

Effective contraceptive methods were defined as intrauterine devices (IUDs) and oral, vaginal, transdermal and implant hormonal contraceptives. Records for oral contraceptives indicating 21-day treatments, transdermal contraceptive patches and similarly for one vaginal ring supply, were treated as 28 days. Records for one implant were treated as 3 years' duration. The period of guaranteed contraception for IUDs was considered to be 5 years (3 years for levonorgestrel $13.5 \mathrm{mg}$ IUDs). In addition, adequate contraception was defined as a healthcare claim indicating an insertion or follow-up for an IUD with a 5 -year duration of coverage since the date of the procedure.

\subsection{Concomitant Use of Contraceptives with Isotretinoin}

As the main outcome, we estimated the proportion of women who used systemic isotretinoin and had a concomitant record of contraceptive use. Concomitant adequate contraceptive coverage was defined as contraception 30 days before isotretinoin treatment started, during and 30 days after the use of isotretinoin ended. Absence of concomitant contraception was defined as no record of contraception in the period of 30 days before, during and 30 days after isotretinoin use. Partial coverage was defined as no evidence of contraception for at least one of the (sub)periods: 30 days before, during or after isotretinoin treatment.

\subsection{Identification of Isotretinoin-exposed Pregnancies}

Diagnostic codes were used to detect pregnancy timing in relation to isotretinoin treatment. Pregnancy was considered to be isotretinoin-exposed when healthcare service data coincided with isotretinoin use, until 60 days after treatment ended. We used 60 days after the end of isotretinoin treatment as the cut-off value for exposed pregnancy because $\sim 88 \%$ of Estonian women are diagnosed as being pregnant within 8-9 weeks of gestation [10].

\subsection{Other Variables}

Specialists who prescribe isotretinoin were identified (dermatologists, family medicine, other), as were the diseases for which isotretinoin was prescribed (seborrhoeic dermatitis, acne, rosacea, other follicular disorders, and other). 


\subsection{Statistical Analysis}

Descriptive statistics summarized patient characteristics, prescribed isotretinoin treatment and use of contraception. Subgroup analyses stratified by age (15-19, 20-29, 30-39, 40-45 years) were performed to account for hypothesized differences in contraceptive use patterns between younger and older women.

We estimated cumulative risk (for isotretinoin-exposed) pregnancy (per 1000 women) together with the $95 \%$ confidence intervals (binomial exact) for the age groups specified above and for women covered, partially covered and not covered by contraception. Statistical differences between age groups were tested by Fisher's exact test. We further estimated incidence rate (per 100 months exposed to isotretinoin) for isotretinoin-exposed pregnancy together with (Poisson) confidence intervals.

Factors associated with contraceptive coverage during isotretinoin treatment were assessed using logistic regression analysis. We generated univariate and multivariable estimates using stepwise regression and present odds ratios together with $95 \%$ confidence estimates.

All statistical analyses were performed using STATA version 14.2.

The study procedures were in accordance with local data protection regulations. The analysis used existing patient records containing only non-identifiable data and was therefore exempt from ethical review.

\section{Results}

\subsection{Characteristics of Study Subjects and Treatment Courses}

Over the study period, a total of 3115 women filled an isotretinoin prescription in Estonia; the majority $(n=2792$, $89.6 \%$ ) were $15-45$ years old (Fig. 1). Among the women aged $15-45$ years, $34.4 \%(n=961)$ were $15-19$ years old, $43.7 \%$ were $20-29$ years old, $17.3 \%$ were $30-39$ years old and 4.6\% ( $n=127)$ were older. From 2012 to 2016, the number of women filling an isotretinoin prescription each year decreased (from 864 women in 2012 to 407 women in 2016, $p=0.07$ ). Isotretinoin prescription intensity decreased significantly from 3.3 to 1.6 per 1000 women $(p<0.001)$. Most prescriptions were issued by dermatologists $(n=2662,95.3 \%)$, followed by family physicians $(n=78,2.8 \%)$.

The mean duration of isotretinoin treatment was 108 days (SD 60, range 30-408 days) and the mean daily dose was $36.6 \mathrm{mg}$ (SD 16.5, range 6.9-210 mg). Isotretinoin was prescribed for acne $(n=2621,93.9 \%)$, infrequently for rosacea $(n=129,4.6 \%)$ and rarely for other conditions (seborrhoeic dermatitis, $n=12$; other follicular diseases, $\mathrm{n}=20$ ).

\subsection{Use and Concomitant Use of Contraceptives}

During the 5-year study period, 1716 women aged 15-45 years used the specified contraceptive methods. Concomitant to isotretinoin use, contraception could be documented for 828 women $(n=647,78.1 \%$ hormonal contraception and $n=181,21.9 \%$ IUD). Among all women receiving isotretinoin, only $15.7 \% \quad(95 \%$ CI 14.4-17.1) had full contraceptive coverage (including 30 days before and 30 days after taking isotretinoin); $13.9 \%$ (95\% CI 12.7-15.3) of women had partial coverage. Full coverage was highest among women over 30 years of age, and partial coverage tended to be more common among women in their twenties and thirties (Table 1).

\subsection{Pregnancies Recorded During and After Treatment with Isotretinoin}

Over the 5-year period of observation, 19.8\% (555/2792) of the women had a healthcare visit during which a pregnancy was recorded. Of these 555 women, ten were pregnant during the isotretinoin treatment-related period under observation defined by the following ICD 10 codes: Z32.1 $(n=3)$, Z33 $(n=2)$, O04.9 $(n=2)$, O00.1 $(n=3)$. These women potentially had isotretinoin-exposed pregnancies. Eight of the women in this analysis were not using contraception while the other two women with potentially exposed pregnancies were either partially or fully covered. One woman received the first isotretinoin prescription 2 months earlier than the prescription for oral contraception (G03AA16) and had a pregnancy termination (ICD 10: O04.9) a month after filling the contraception prescription. The second woman had an IUD placed 34 months before starting isotretinoin and had a diagnosis of ectopic pregnancy (ICD 10: O00.1) 6 weeks after filling the last isotretinoin prescription. The risk for exposed pregnancy was 3.6 (95\% CI 2.0-7.0) per 1000 treated women (10/2792) over the 5-year observation period. The rates varied substantially when stratified by age: 7.9 (95\% CI $0.2-43.1)$ per 1000 women aged 40-45 years (1/127), 4.1 (95\% CI $0.5-14.8)$ per 1000 in women aged 30-39 (2/484), 4.9 (95\% CI 1.8-10.7) (per 1000 in women aged 20-29 (6/ $1220)$, and 1.0 (95\% CI 0.3-5.8) per 1000 in women aged $15-19$ years $(1 / 961)(\mathrm{p}=0.228)$.

The risk for exposed pregnancy did not differ between women considered to be adequately covered $(1 / 439 ; 2.3$ per 1000 women, $95 \%$ CI $0.1-12.6)$, partially covered (1/389; 2.6 per 1000 women, $95 \%$ CI $0.1-14.2$ ) or not covered (8/ 1964 ; 4.1 per 1000 women, $95 \%$ CI $1.8-8.0)$ with contraception during isotretinoin treatment $(p=0.99)$. 


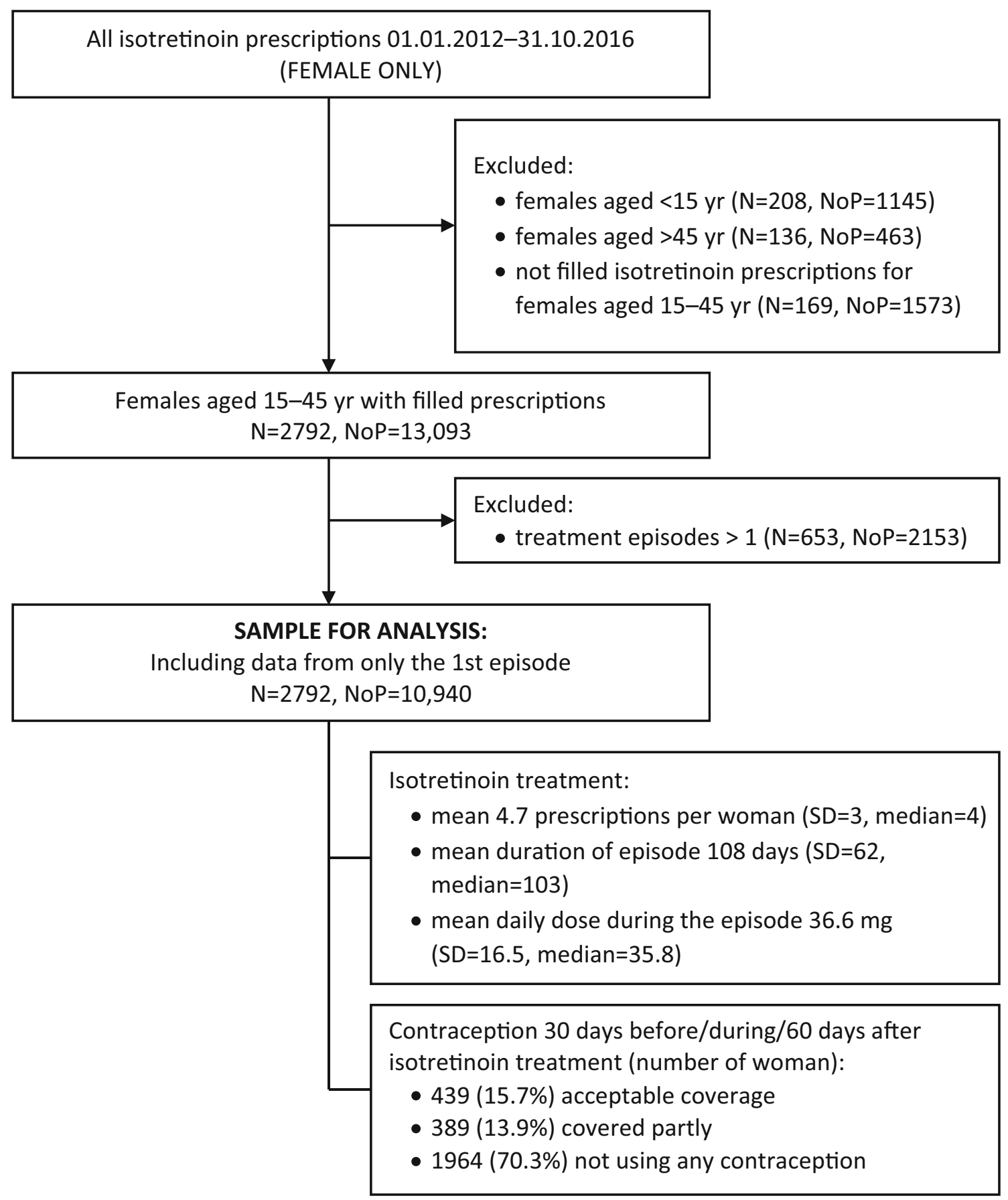

Fig. 1 Flow chart showing the data selection process for the analysis of the compliance with pregnancy prevention recommendations for isotretinoin in Estonia (2012-2016). $N$ number of individuals, $N o P$ number of prescriptions

To describe the factors associated with adequate contraception coverage during isotretinoin treatment, we compared women with full coverage to those partially or not covered. The incidence rate for isotretinoin-exposed pregnancy among women adequately covered was 0.047 per 100 months (95\% CI 0.007-0.336) on isotretinoin and 0.081 per 100 months (95\% CI 0.042-0.155) among women partially/not covered $(p=0.611)$.
Factors associated with receiving adequate contraceptive coverage during isotretinoin treatment are presented in Table 2. After adjustment, three factors remained significantly associated with odds of coverage. Being covered with contraception increased with the age of the woman: the youngest women had the lowest odds for being covered (adjusted OR 0.19, 95\% CI 0-0.27) in comparison to the highest odds among women aged 30-39 years (adjusted 
Table 1 Concomitant use of contraceptives by women aged 15-45 years receiving isotretinoin treatment, Estonia, 2012-2016

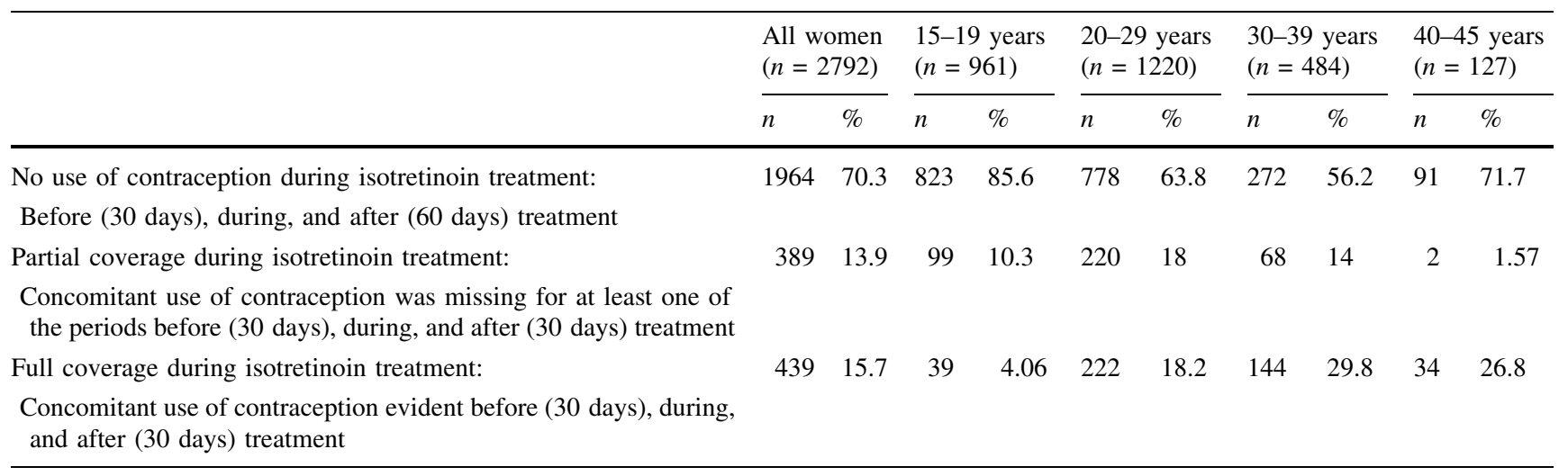

Table 2 Factors associated with receiving adequate contraceptive coverage during isotretinoin treatment among women aged 15-45 years, Estonia, 2012-2016

\begin{tabular}{|c|c|c|c|c|c|c|c|c|c|c|c|}
\hline & \multirow{2}{*}{$\begin{array}{l}\text { Not/partially } \\
\text { covered with } \\
\text { contraception }\end{array}$} & \multirow{2}{*}{$\begin{array}{l}\text { Covered with } \\
\text { contraception }\end{array}$} & \multirow{2}{*}{$\begin{array}{l}\text { Proportion }(\%) \\
\text { of those fully } \\
\text { covered }\end{array}$} & \multicolumn{4}{|c|}{ Univariate models } & \multicolumn{4}{|c|}{ Adjusted model } \\
\hline & & & & OR & $95 \%$ & & $p$ value & OR & $95 \%$ & & $p$ value \\
\hline \multicolumn{12}{|l|}{ Age (years) } \\
\hline $15-19$ & 922 & 39 & 4.1 & 0.19 & 0 & 0.27 & $\mathbf{0}$ & 0.18 & 0.13 & 0.26 & $\mathbf{0}$ \\
\hline $20-29$ & 998 & 222 & 18.2 & 1 & & & & 1 & & & \\
\hline $30-39$ & 340 & 144 & 29.8 & 1.90 & 0 & 2.428 & $\mathbf{0}$ & 1.94 & 1.52 & 2.48 & $\mathbf{0}$ \\
\hline $40-45$ & 93 & 34 & 26.8 & 1.64 & 0 & 2.499 & $\mathbf{0}$ & 1.70 & 1.11 & 2.60 & 0.014 \\
\hline \multicolumn{12}{|l|}{ Diagnosis } \\
\hline Acne & 2228 & 393 & 15.0 & 1 & & & & & & & \\
\hline Other & 125 & 46 & 26.9 & 2.09 & 1.46 & 2.97 & $\mathbf{0}$ & & & & \\
\hline \multicolumn{12}{|c|}{ Specialty of prescribing physician } \\
\hline Dermatovenerologist & 2249 & 413 & 15.5 & 1 & & & & & & & \\
\hline Other & 39 & 13 & 25.0 & 1.82 & 0.96 & 3.43 & 0.066 & & & & \\
\hline Family physician & 65 & 13 & 16.7 & 1.09 & 0.59 & 1.99 & 0.782 & & & & \\
\hline \multicolumn{12}{|c|}{ Mean daily dose of isotretinoin (SD) } \\
\hline & 36.8 & 35.3 & & 0.99 & 0.99 & 1.00 & 0.067 & & & & \\
\hline & 16.3 & 17.3 & & & & & & & & & \\
\hline \multicolumn{12}{|c|}{ The year of treatment episode (beginning) } \\
\hline 2012 & 760 & 104 & 12.0 & 1 & & & & 1 & & & \\
\hline 2013 & 466 & 85 & 15.4 & 1.33 & 0.98 & 1.82 & 0.068 & 1.35 & 0.98 & 1.85 & 0.07 \\
\hline 2014 & 392 & 77 & 16.4 & 1.44 & 1.04 & 1.97 & 0.026 & 1.45 & 1.04 & 2.02 & 0.03 \\
\hline 2015 & 403 & 98 & 19.6 & 1.78 & 1.31 & 2.40 & 0 & 1.78 & 1.30 & 2.44 & 0.00 \\
\hline 2016 & 332 & 75 & 18.4 & 1.65 & 1.19 & 2.28 & 0.002 & 2.03 & 1.44 & 2.86 & 0.00 \\
\hline \multicolumn{12}{|c|}{ Mean duration of the treatment episode (months) (SD) } \\
\hline & 4.6 & 4.7 & & 1.02 & 0.97 & 1.07 & 0.363 & 1.07 & 1.02 & 1.13 & 0.01 \\
\hline & 2.0 & 2.1 & & & & & & & & & \\
\hline
\end{tabular}

Statistically significant values are in bold

OR 1.9, 95\% CI 1.5-2.5); and with an increasing length of treatment (adjusted OR 1.07, 95\% CI 1.0-1.1 for a 1-month increase). We also saw the period/time effect with odds of coverage increasing during the period under observation.

\section{Discussion}

This study of national data from Estonia documented very low coverage of adequate contraception during isotretinoin treatment among women of reproductive age. For the 
majority of women $(70.3 \%)$, we were unable to detect any record of contraceptive use concomitant to isotretinoin treatment, and $13.9 \%$ of women were only partially covered and therefore at risk for the teratogenic effects of the drug.

In our study, effective contraception coverage increased with the age of the patient, duration of treatment and period of observation. Low coverage among women aged 15-19 years may suggest many were not yet sexually active. Unlike some other medicines with strict pregnancy contraindications (thalidomide, valproate), the treatment course is shorter and usually has a predictable duration. Women with longer isotretinoin treatment courses were more likely to be covered with effective contraception, which was clearly a positive sign.

An interesting observation in our study was the annualised risk of unintended pregnancies (analogue to the Pearl index) in the group of women partially or not covered by contraception, 0.97 (derived from the risk estimate of potentially exposed pregnancies of $0.081 / 100$ months). This estimate is comparable with that of combined hormonal contraceptives or copper-IUD Pearl indexes [11]. This finding might be explained by the high proportion of women on isotretinoin who are not sexually active, or choose to be abstinent while on isotretinoin [12] in addition to using contraceptive methods not captured in our analysis (i.e. condoms).

To our knowledge, few studies have assessed compliance with recommended contraceptive use according to official pregnancy prevention programs. Prevalence estimates for contraception during isotretinoin vary from about one-third (Canada [7], USA [5]) to half (Netherlands [3, 6]) to above $80 \%$ (USA [14]). In the US study, the authors concluded that concomitant contraception was less common for younger than older women [5], but no similar trend was observed in the Netherlands [3]. The rate of contraception in our study (15.7\%) appears to be about half of the lowest published estimate $[5,7]$.

While these estimates of contraceptive coverage vary widely, the rates of isotretinoin-exposed pregnancies are quite constant, varying between 2 and 6 per 1000 treated women (4-6/1000 in Canada [7], 2.7-3.5/1000 in the USA [12-14]. Interestingly, there is no clear association between contraception coverage rates and exposed pregnancy incidence. This discrepancy may be partially explained by methodological differences in studies, and it is difficult to estimate patient adherence to prescribed treatment regimens. One remaining consideration that has not been elucidated by research to date is the proportion of women taking isotretinoin who choose abstinence, and thus do not need contraception. We acknowledge that while these (young) women may not be sexually active upon beginning treatment the situation is likely very unpredictable.
However, recommending prophylactic contraception would obviously require careful economic, clinical and socioemotional consideration, and potentially warrants a more individualised approach [15].

Risk-minimization measures are an essential part of the life-cycle management of a drug [16]. Oral isotretinoin is a treatment of choice (with no meaningful alternative) for many patients with severe acne. Whether instituting increasingly restrictive programs is the optimal approach warrants further evidence [17]. Importantly, enhanced measures such as PPP in the EU or iPledge in the USA pose an extra burden on the health system and patients, and thus should be carefully justified and monitored to ascertain whether added value is expected [18]. The current literature may not justify monthly visits during isotretinoin use, nor using two different contraceptive methods. Furthermore, the requirements imposed on physicians and patients must align with the existing regulations. For example, Estonian prescription regulations [9] requiring at least 2 months of treatment create an inherent conflict between PPP requirements and local laws, leaving physicians in a potentially vulnerable position.

Based on our results we speculate that filing information on pregnancies occurring during isotretinoin treatment is suboptimal even though the PPP highlights the importance of reporting on exposed pregnancies. Therefore, essential information for marketing authorisation holders and competent authorities is missing, and this may lead to the false conclusion that PPP is effective in eliminating the risk of pregnancy during isotretinoin exposure.

The limitations of administrative data apply here as well. It is possible that we may have underestimated the proportion of women covered with effective contraception, and we may have missed other effective contraceptive methods like sterilization or infertility. This misclassification is unlikely to be substantial because sterilization among women younger than 44 years is very rare in Estonia: $<1 \%$ [18]. Per approved labelling of the drug, appropriate birth control assumes parallel use of two methods of birth control. We have no information on dual contraceptive coverage, especially for condoms (the method of choice among one in three Estonian women) [19]. In addition, while we might have missed some concomitant use of previously inserted intrauterine contraceptives, we did capture data for health services related to monitoring and control of IUDs, most likely capturing some of these women. To minimize mis-classification bias, we limited the analysis to treatment periods defined by pharmacy prescription fill dates. Still, we cannot be sure that patients for whom the drug was dispensed actually took it.

Finally, the number of pregnancies potentially exposed to isotretinoin was small and derived estimates are 
therefore imprecise. Our study period captured pregnancies up to 60 days after discontinuation of isotretinoin. The overwhelming majority of women in Estonia are diagnosed with pregnancy by gestational week 9, thus some potentially exposed pregnancies may have been missed. While these biases may slightly influence estimates of coverage and exposed pregnancies, they seem unlikely to have caused the clear patterns observed in this study. Strengths of the study include a true nationwide, population-based analysis capturing extensive administrative and utilization data for Estonian women.

\section{Conclusion}

Our study adds to the existing literature documenting the limited effects of PPP. Obviously, it is imperative to provide oral isotretinoin to patients in the safest manner possible. The effectiveness of PPP at the population level, and thus the cost-effectiveness of enhanced isotretinoin PPP, remains unclear. A rigorous program assessment is necessary to identify whether new measures should be taken or whether weaknesses in policy or implementation can be corrected.

\section{Compliance with Ethical Standards}

Funding This work was supported grant \# IUT34-17 from the Estonian Ministry of Education and Research

Conflicts of interest $\mathrm{AU}, \mathrm{HP}, \mathrm{KK}, \mathrm{OL}, \mathrm{ML}$, and $\mathrm{MU}$ report no conflicts of interest.

Ethics approval The study procedures were in accordance with local data protection regulations. The analysis used existing patient records containing only non-identifiable data and was therefore exempt from ethical review.

Open Access This article is distributed under the terms of the Creative Commons Attribution-NonCommercial 4.0 International License (http://creativecommons.org/licenses/by-nc/4.0/), which permits any noncommercial use, distribution, and reproduction in any medium, provided you give appropriate credit to the original author(s) and the source, provide a link to the Creative Commons license, and indicate if changes were made.

\section{References}

1. Goodfield MJ, Cox NH, Bowser A, et al. Advice on the safe introduction and continued use of isotretinoin in acne in the U.K. 2010. Br J Dermatol. 2010;162(6):1172-9.

2. Commission decision background information on Article 29 referral isotretinoin 2003. http://www.ema.europa.eu/docs/en_ GB/document_library/Referrals_document/Isotretinoin_29/ WC500010881.pdf. Accessed 25 Jul 2017.
3. Crijns I, Straus S, Luteijn M, et al. Implementation of the harmonized EU isotretinoin Pregnancy Prevention Programme: a questionnaire survey among European regulatory agencies. Drug Saf. 2012;35(1):27-32.

4. Crijns HJ, Straus SM, Gispen-de Wied C, et al. Compliance with pregnancy prevention programmes of isotretinoin in Europe: a systematic review. Br J Dermatol. 2011;164(2):238-44.

5. Pinheiro SP, Kang EM, Kim CY, et al. Concomitant use of isotretinoin and contraceptives before and after iPledge in the United States. Pharmacoepidemiol Drug Saf. 2013;22(12):1251-7.

6. Teichert M, Visser LE, Dufour M, et al. Isotretinoin use and compliance with the Dutch Pregnancy Prevention programme: a retrospective cohort study in females of reproductive age using pharmacy dispensing data. Drug Saf. 2010;33(4):315-26.

7. Henry D, Dormuth C, Winquist B, et al. Occurrence of pregnancy and pregnancy outcomes during isotretinoin therapy. CMAJ. 2016;188(10):723-30.

8. Estonian health insurance fund (2017). https://www.haigekassa. ee/et/haigekassa/statistika/ravikindlustus. Accessed 26 Nov 2017.

9. Conditions and procedure for preparation, dividing-up and checking of medicinal products in pharmacies, a list of medicinal products prepared as officinal formulae in pharmacies (2014). https://www.riigiteataja.ee/en/eli/ee/MHL/reg/528032016009/ consolide. Accessed 27 Nov 2017.

10. Estonian Medical Birth Registry. Estonian Abortion Registry. http://www.tai.ee/et/tegevused/registrid/meditsiinilinesunniregister-ja-raseduskatkestus-andmekogu/statistika. Accessed 23 Nov 2017.

11. World Health Organization 2015. Medical eligibility criteria for contraceptive use, 5th ed. http://apps.who.int/iris/bitstream/ 10665/181468/1/9789241549158_eng.pdf?ua=1. Accessed 27 Nov 2017.

12. Shin J, Cheetham TC, Wong L, et al. The impact of the iPLEDGE program on isotretinoin fetal exposure in an integrated health care system. J Am Acad Dermatol. 2011;65(6):1117-25.

13. Mitchell AA, Van Bennekom CM, Louik C. A pregnancy-prevention program in women of childbearing age receiving isotretinoin. N Engl J Med. 1995;333(2):101-6.

14. Brinker A, Kornegay C, Nourjah P. Trends in adherence to a revised risk management program designed to decrease or eliminate isotretinoin-exposed pregnancies: evaluation of the accutane SMART program. Arch Dermatol. 2005;141(5):563-9.

15. Pierson JC, Ferris LK, Schwarz EB. We pledge to change iPLEDGE. JAMA Dermatol. 2015;151(7):701-2.

16. European Medicine Agency 2012. Guideline on good pharmacovigilance practices (GVP) Module V-Risk management systems. http://www.ema.europa.eu/docs/en_GB/document_library/ Scientific_guideline/2012/06/WC500129134.pdf. Accessed 27 Nov 2017.

17. Thiboutot D, Gollnick H, Bettoli V, et al. Oral isotretinoin and pregnancy prevention programmes. $\mathrm{Br} \quad \mathrm{J}$ Dermatol. 2012;166:466-7.

18. Zomerdijk IM, Ruiter R, Houweling LM, et al. Isotretinoin exposure during pregnancy: a population-based study in The Netherlands. BMJ Open. 2014;4(11):e005602.

19. Lippus H, Laanpere M, Part Ket al. Estonian Women's Health 2014: sexual and reproductive health, health behaviour, attitudes and use of healthcare services. Survey report. Tartu; 2015. https:// sisu.ut.ee/sites/default/files/naisteterviseuuring/files/ uusestre2014_loppraport.pdf. Accessed 27 Nov 2017. 